\title{
Healthcare Encounters Start Timepoint
}

National Cancer Institute

\section{Source}

National Cancer Institute. Healthcare Encounters Start Timepoint. NCI Thesaurus. Code C124022.

A point in time that indicates the beginning of a healthcare encounter. 